\title{
Highly deuterated pre-stellar cores in a high-mass star formation region ${ }^{\star}$
}

\author{
F. Fontani ${ }^{1}$, P. Caselli ${ }^{2,3}$, T. L. Bourke ${ }^{4}$, R. Cesaroni ${ }^{2}$, and J. Brand ${ }^{1}$ \\ 1 INAF - Istituto di Radioastronomia, via Gobetti 101, 40129 Bologna, Italy \\ e-mail: ffontani@ira.inaf.it \\ 2 INAF - Osservatorio Astrofisico di Arcetri, Largo E. Fermi 5, 50125 Firenze, Italy \\ School of Physics and Astrophysics, University of Leeds, Leeds LS2 9JT, UK \\ ${ }^{4}$ Harvard-Smithsonian Center for Astrophysics, 60 Garden Street MS42, Cambridge, MA 02138, USA
}

Received 31 October 2007 / Accepted 26 November 2007

\section{ABSTRACT}

\begin{abstract}
Aims. We have observed the deuterated gas in the high-mass star formation region IRAS $05345+3157$ at high-angular resolution, in order to determine the morphology and the nature of this gas.

Methods. We have mapped the $\mathrm{N}_{2} \mathrm{H}^{+}(1-0)$ line with the Plateau de Bure Interferometer, and the $\mathrm{N}_{2} \mathrm{D}^{+}(3-2)$ and $\mathrm{N}_{2} \mathrm{H}^{+}(3-2)$ lines with the Submillimeter Array.

Results. We have detected two condensations in $\mathrm{N}_{2} \mathrm{D}^{+}$, with masses of $\sim 2-3$ and $\sim 9 M_{\odot}$ and diameters of 0.05 and 0.09 pc, respectively. The high deuterium fractionation $(\sim 0.1)$ and the line parameters of the $\mathrm{N}_{2} \mathrm{D}^{+}$condensations indicate that they are probably low- to intermediate-mass pre-stellar cores, even though other scenarios are possible.
\end{abstract}

Key words. stars: formation - radio lines: ISM - ISM: individual: IRAS 05345+3157 - ISM: molecules

\section{Introduction}

The initial conditions of the star formation process are still a matter of debate. Studies have begun to unveil the chemical and physical properties of starless low-mass cores on the verge of forming low-mass stars (Kuiper et al. 1996; Caselli et al. 2002a,b; Tafalla et al. 2002, 2006), demonstrating that in the dense and cold nuclei of these cores, C-bearing molecular species such as CO and CS are strongly depleted (e.g. Caselli et al. 2002b; Tafalla et al. 2002), while N-bearing molecular ions such as $\mathrm{N}_{2} \mathrm{H}^{+}$and $\mathrm{N}_{2} \mathrm{D}^{+}$(Caselli et al. 2002a,b; Crapsi et al. 2005) maintain large abundances in the gas phase, and their column density ratios reach values of $\sim 0.1$ or more, much higher than the cosmic $[\mathrm{D} / \mathrm{H}]$ elemental abundance $\left(\sim 10^{-5}\right.$, Oliveira et al. 2003).

The characterisation of the earliest stages of the formation process of high-mass stars is more difficult than for low-mass objects, given their shorter evolutionary timescales, larger distances, and strong interaction with their environments. To check whether the chemical properties peculiar to the earliest stages of low-mass stars are also valid for high-mass stars, Fontani et al. (2006) observed the deuterated gas in 10 sources selected from two samples of high-mass protostar candidates (Molinari et al. 1996; Sridharan et al. 2002), which are believed to be the closest to the earliest stages of the high-mass star formation process. One of these, IRAS 05345+3157 (hereafter I05345), stands out because of its very interesting characteristics: it is a luminous $\left(1.38 \times 10^{3} L_{\odot}\right.$ at a distance of $1.8 \mathrm{kpc}$, Zhang et al. 2005) young stellar object, embedded in a massive $\left(\sim 180 M_{\odot}\right)$ dusty

^ Based on observations carried out with the IRAM Plateau de Bure Interferometer. IRAM is supported by INSU/CNRS (France), MPG (Germany) and IGN (Spain). clump (Fontani et al. 2006) in which Molinari et al. (2002) revealed a complex structure in the molecular gas observed in the $\mathrm{HCO}^{+}(1-0)$ line. From IRAM-30m spectra of the $\mathrm{N}_{2} \mathrm{H}^{+}(1-0)$ and $\mathrm{N}_{2} \mathrm{D}^{+}$(2-1) lines, Fontani et al. (2006) have derived an average deuterium fractionation of $\sim 0.01$, quite close to the values found by Crapsi et al. (2005) in low-mass starless cores. These results indicate the presence in the source of molecular gas with physical conditions similar to those of low-mass starless cores (i.e. $T \sim 10 \mathrm{~K}$ and $n_{\mathrm{H}_{2}} \sim 10^{6} \mathrm{~cm}^{-3}$ ). However, the precise location and the distribution of this gas, required to understand its nature, can be determined only through higher angular resolution observations.

In this letter, we present observations of $\mathrm{N}_{2} \mathrm{H}^{+}$and $\mathrm{N}_{2} \mathrm{D}^{+}$ towards I05345, obtained with the Submillimeter Array (SMA) and the Plateau de Bure Interferometer (PdBI), and we report on the detection of two compact condensations of $\mathrm{N}_{2} \mathrm{D}^{+}$that have chemical features typical of low-mass pre-stellar core candidates. A full report and a more detailed analysis of the data obtained will be presented in a forthcoming paper.

\section{Observations and data reduction}

Observations of $\mathrm{N}_{2} \mathrm{D}^{+}(3-2)$ (at $231321 \mathrm{MHz}$ ) and $\mathrm{N}_{2} \mathrm{H}^{+}$(3-2) (at $279511.7 \mathrm{MHz}$ ) towards $\mathrm{I} 05345$ were carried out with the $\mathrm{SMA}^{1}$ (Ho et al. 2004) in the compact configuration on 30 January and 21 February 2007, respectively. The correlator was configured to observe the continuum emission and several

\footnotetext{
1 The Submillimeter Array is a joint project between the Smithsonian Astrophysical Observatory and the Academia Sinica Institute of Astronomy and Astrophysics, and is funded by the Smithsonian Institution and the Academia Sinica.
} 


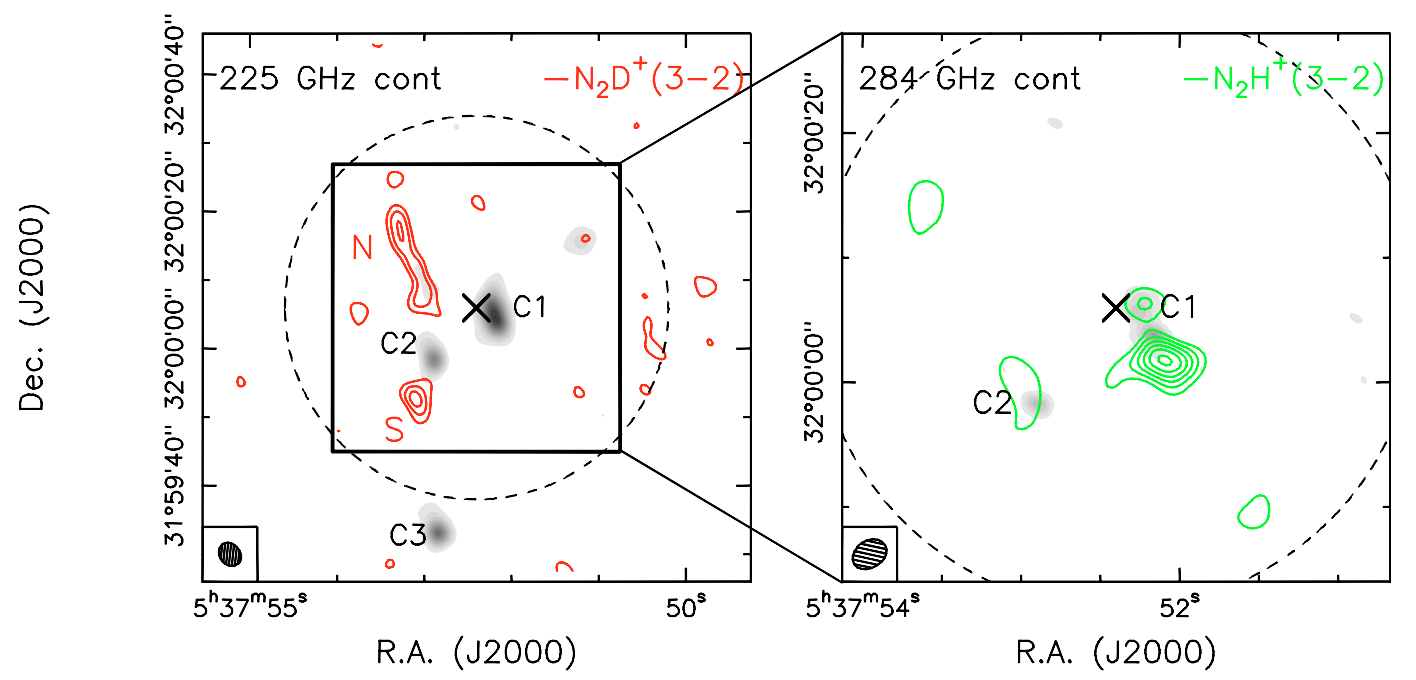

Fig. 1. Left panel: map of the emission of the $\mathrm{N}_{2} \mathrm{D}^{+}(3-2)$ line integrated between -18.37 and $-16.7 \mathrm{~km} \mathrm{~s}^{-1}$, obtained with the SMA towards I05345 (red contours). The two main condensations are indicated as $\mathrm{N}$ and S. Contour levels start from the $3 \sigma \mathrm{rms}\left(\sim 0.09 \mathrm{Jy} \mathrm{beam}^{-1}\right)$, and are in steps of $2 \sigma$. The grey scale represents the $225 \mathrm{GHz}$ continuum (first level is the $3 \sigma \mathrm{rms}=0.006 \mathrm{Jy} \mathrm{beam}^{-1}$; step $=3 \sigma \mathrm{rms}$ ). The three compact continuum cores are indicated as C1, C2 and C3. The cross corresponds to the map centre (I05345 nominal position) and the dashed circle represents the SMA primary beam at $225 \mathrm{GHz}\left(\sim 56^{\prime \prime}\right)$. The ellipse in the bottom left corner shows the synthesised beam of the $\mathrm{N}_{2} \mathrm{D}^{+}$image.

Right panel: map of the $\mathrm{N}_{2} \mathrm{H}^{+}$(3-2) line emission integrated between -19.6 and $-16.1 \mathrm{~km} \mathrm{~s}^{-1}$ towards I05345 (green contours), observed with the SMA (first contour $=3 \sigma \mathrm{rms}\left[\sim 0.45 \mathrm{Jy}_{\text {beam }}^{-1}\right]$; step $=2 \sigma \mathrm{rms}$ ). The grey scale represents the $284 \mathrm{GHz}$ continuum (first level is the $3 \sigma$ $\mathrm{rms}=0.018 \mathrm{Jy} \mathrm{beam}^{-1}$; step $\left.=3 \sigma \mathrm{rms}\right)$. The dashed circle represents the primary beam at $284 \mathrm{GHz}\left(\sim 44^{\prime \prime}\right)$. The cross indicates the map centre and the ellipse in the bottom left corner is the SMA synthesised beam of the $\mathrm{N}_{2} \mathrm{H}^{+}$image.

other molecular lines simultaneously. The phase centre was the nominal position of the sub-mm peak detected with the JCMT (Fontani et al. 2006), namely $\mathrm{RA}(\mathrm{J} 2000)=05^{\mathrm{h}} 37^{\mathrm{m}} 52.4^{\mathrm{s}}$ and $\operatorname{Dec}(\mathrm{J} 2000)=32^{\circ} 00^{\prime} 06^{\prime \prime}$, and the local standard of rest velocity $V_{\mathrm{LSR}}$ is $-18.4 \mathrm{~km} \mathrm{~s}^{-1}$. For gain calibrations, observations of I05345 were alternated with the sources 3C 111 and J0530+135. 3C 279 and Callisto were used for passband and flux calibration, respectively. The SMA data were calibrated with the MIR package (Qi 2005), and imaged with MIRIAD (Sault et al. 1995). Channel maps were created with natural weighting, attaining a resolution of: 3 !' $7 \times 3$ !' 0 for the $\mathrm{N}_{2} \mathrm{D}^{+}(3-2)$ channel map; 3 ." $0 \times 2$." 8 for the $225 \mathrm{GHz}$ continuum image; 2 ." $7 \times 2$.' 0 for the $\mathrm{N}_{2} \mathrm{H}^{+}$(3-2) channel map; 1 ." $9 \times 1$." 2 for the $284 \mathrm{GHz}$ continuum image.

We observed the $\mathrm{N}_{2} \mathrm{H}^{+}$(1-0) line at $93173.7725 \mathrm{MHz}$ towards I05345 with the PdBI on 11 and 20 August 2006, in the D configuration, and on 3 April 2007 in the C configuration. We used the same phase reference and $V_{\mathrm{LSR}}$ velocities as for the SMA observations. The nearby point sources $0507+179$ and $0552+398$ were used as phase calibrators, while bandpass and flux scale were calibrated from observations of 3C 345 and MWC349, respectively. For continuum measurements, we placed two $320 \mathrm{MHz}$ correlator units in the band when making the observations in D configuration, and six $320 \mathrm{MHz}$ correlator units in $\mathrm{C}$ configuration. The $\mathrm{N}_{2} \mathrm{H}^{+}$lines were excluded in averaging these units to produce the final continuum image (at $\sim 96095 \mathrm{MHz}$ ). The synthesised beam size of the $\mathrm{N}_{2} \mathrm{H}^{+}$channel map was 3.' $2 \times 3$." 4 , while that of the continuum was 3 ." $1 \times 3$ ". 2 . We stress that the observations of the $\mathrm{N}_{2} \mathrm{H}^{+}(1-0)$ and $\mathrm{N}_{2} \mathrm{D}^{+}$ (3-2) lines have approximately the same angular resolution. The data have been reduced with the GILDAS software, developed at IRAM and the Observatoire de Grenoble.

The spectra of both $\mathrm{N}_{2} \mathrm{H}^{+}$and $\mathrm{N}_{2} \mathrm{D}^{+}$obtained from the cleaned maps have been analysed with the software CLASS, with the method described in Sect. 2.1.1 of Fontani et al. (2006).

\section{Results}

\subsection{Structure of the source}

The map of the integrated intensity of the $\mathrm{N}_{2} \mathrm{D}^{+}$(3-2) line, superimposed on the $225 \mathrm{GHz}$ continuum map, is shown in the left panel of Fig. 1. The map indicates that the $\mathrm{N}_{2} \mathrm{D}^{+}$emission arises from two molecular condensations: an extended clump located $\sim 10^{\prime \prime} \mathrm{N}-\mathrm{E}$ of the map centre and elongated $\sim 15^{\prime \prime}$ in the $\mathrm{N}-\mathrm{S}$ direction, and a compact core, $\sim 5^{\prime \prime}$ in size, located $\sim 15^{\prime \prime}$ $\mathrm{S}-\mathrm{E}$ of the map centre. In the following Sections, we will identify these condensations as $\mathrm{N}$ and $\mathrm{S}$, respectively, and they represent the targets of the present study. The $225 \mathrm{GHz}$ continuum image shows two main compact cores inside the SMA primary beam, one approximately corresponding to the map centre and the other located $\sim 10^{\prime \prime} \mathrm{S}-\mathrm{E}$ of the map centre. Another compact source outside the interferometer primary beam is located $\sim 35^{\prime \prime}$ south of the map centre, and it corresponds to the southern continuum source detected in the JCMT image by Fontani et al. (2006, see their Fig. A.3). We will call these cores C1, C2 and $\mathrm{C} 3$, respectively. None of these cores overlaps with the two $\mathrm{N}_{2} \mathrm{D}^{+}$condensations. Some extended emission, however, is detected at $\sim 3 \sigma$ towards $\mathrm{N}$. The right panel of Fig. 1 shows the $\mathrm{N}_{2} \mathrm{H}^{+}$(3-2) integrated emission superimposed on the $284 \mathrm{GHz}$ continuum emission, both observed with the SMA. At this frequency the continuum source $\mathrm{C} 1$ is resolved into a main peak and a fainter secondary peak north of the main one. The $\mathrm{N}_{2} \mathrm{H}^{+}$ (3-2) line integrated emission is compact and overlaps well with core $\mathrm{C} 1$, while it is detected only at $3 \sigma$ towards $\mathrm{C} 2$, and is undetected towards $\mathrm{N}$ and $\mathrm{S}$. The detection of $\mathrm{N}_{2} \mathrm{H}^{+}$towards $\mathrm{C} 1$, and the non-detection of $\mathrm{N}_{2} \mathrm{D}^{+}$at that location, is probably due to the continuum image being dominated by the emission of the warm dust, so that in the continuum condensation the temperature is too high for significant deuterium fractionation.

The integrated intensity map of the main group of the hyperfine (hfs) components of the $\mathrm{N}_{2} \mathrm{H}^{+}(1-0)$ line (see e.g. Caselli et al. 1995) observed with the PdBI is shown in Fig. 2. The 


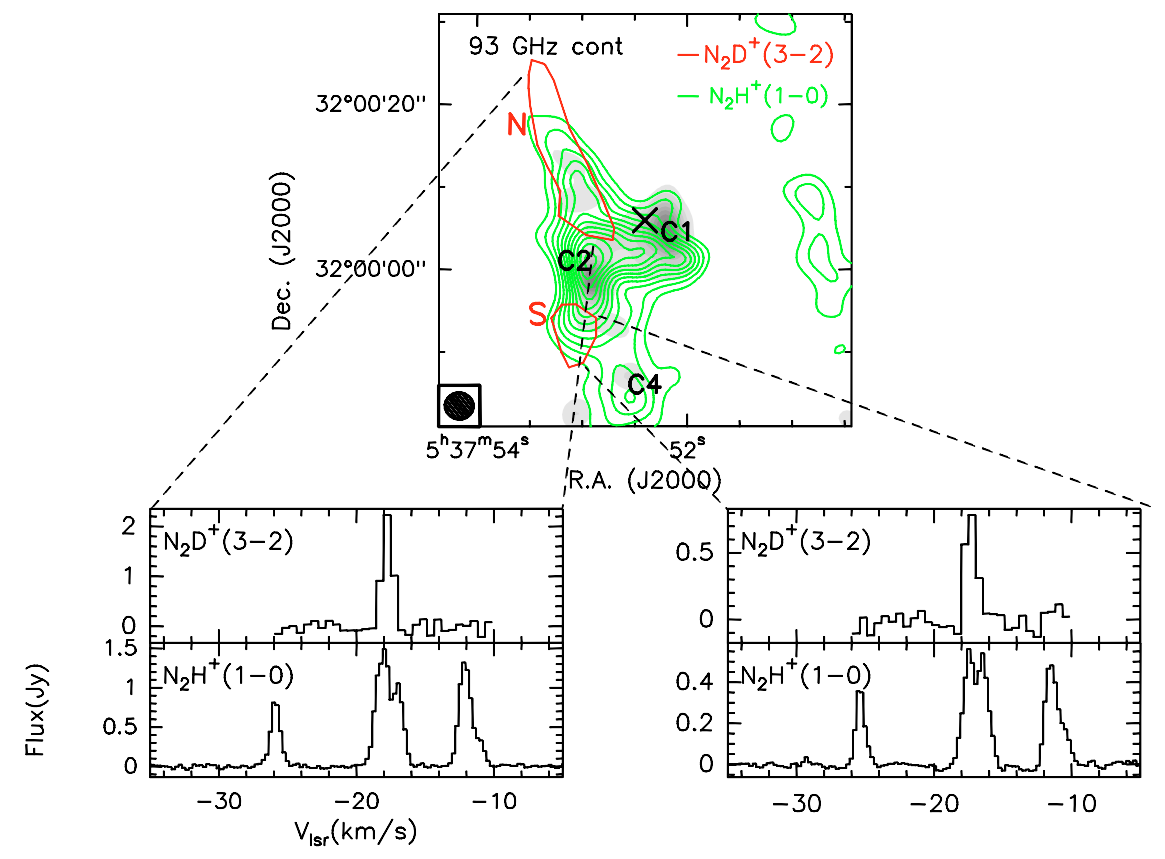

Fig. 2. Map of the intensity of the $\mathrm{N}_{2} \mathrm{H}^{+}(1-0)$ line (green contours) integrated between -21.2 and $-14.5 \mathrm{~km} \mathrm{~s}^{-1}$, corresponding to the main group of the hyperfine components, observed with the PdBI. Levels range from the $3 \sigma \mathrm{rms}$, which is $\sim 0.02 \mathrm{Jy}^{\text {beam }}{ }^{-1}$, to $0.26 \mathrm{Jy} \mathrm{beam}^{-1}$, in steps of $3 \sigma$. The grey scale represents the $97 \mathrm{GHz}$ continuum: the levels range from the $3 \sigma \mathrm{rms}\left(4.2 \times 10^{-4} \mathrm{Jy} \mathrm{beam}^{-1}\right)$ to $4 \times 10^{-3} \mathrm{Jy}_{\text {beam }}^{-1}$, in steps of $3 \sigma$. Below the map, the spectra of $\mathrm{N}_{2} \mathrm{D}^{+}(3-2)$ and $\mathrm{N}_{2} \mathrm{H}^{+}(1-0)$ integrated over the red contours, which correspond to the $3 \sigma$ level of the $\mathrm{N}_{2} \mathrm{D}^{+}$ condensations $\mathrm{N}$ and $\mathrm{S}$ (see Fig. 1), are shown. The other symbols have the same meaning as in the left panel of Fig. 1. The continuum source identified in Fig. 1 as $\mathrm{C} 3$ is out of the box.

Table 1. $\mathrm{N}_{2} \mathrm{H}^{+}(1-0)$ and $\mathrm{N}_{2} \mathrm{D}^{+}(3-2)$ line parameters. Between parentheses, the uncertainties of the fitting procedure (see text) are given.

\begin{tabular}{|c|c|c|c|c|c|c|c|}
\hline & $\begin{array}{c}\text { Spectral resolution } \\
\left(\mathrm{km} \mathrm{s}^{-1}\right)\end{array}$ & $\begin{array}{c}\text { Velocity range } \\
\left(\mathrm{km} \mathrm{s}^{-1}\right)\end{array}$ & $\int_{\left(\mathrm{K} \mathrm{km} \mathrm{s}^{-1}\right)} T_{\mathrm{MB}} \mathrm{dV}$ & $\begin{array}{c}V_{\mathrm{LSR}} \\
\left(\mathrm{km} \mathrm{s}^{-1}\right)\end{array}$ & $\begin{array}{c}F W H M \\
\left(\mathrm{~km} \mathrm{~s}^{-1}\right)\end{array}$ & $\tau_{\mathrm{m}}$ & $\begin{array}{l}T_{\mathrm{ex}} \\
(\mathrm{K}) \\
\end{array}$ \\
\hline $\mathrm{N}$ & & & & & & & \\
\hline $\mathrm{N}_{2} \mathrm{H}^{+}(1-0)$ & 0.25 & $-29.9 ;-12.5$ & $8.7(0.3)$ & $-17.915(0.003)$ & $0.732(0.007)$ & $4.132(0.212)$ & $6.7(0.1)$ \\
\hline $\begin{array}{c}\mathrm{N}_{2} \mathrm{D}^{+}(3-2) \\
\mathrm{S}\end{array}$ & 0.5 & $-18.3 ;-16.7$ & $0.64(0.03)$ & $-17.91(0.02)$ & $0.88(0.014)$ & 0.1 (fixed) & $6.7^{1}$ \\
\hline $\mathrm{N}_{2} \mathrm{H}^{+}(1-0)$ & 0.25 & $-26.5 ;-9.3$ & $10.3(0.5)$ & $-17.283(0.005)$ & $0.73(0.01)$ & $6.1(0.4)$ & $6.6(0.1)$ \\
\hline $\mathrm{N}_{2} \mathrm{D}^{+}(3-2)$ & 0.5 & $-18.3 ;-16.7$ & $0.82(0.06)$ & $-17.51(0.04)$ & $0.88(0.06)$ & 0.1 (fixed) & $6.6^{1}$ \\
\hline
\end{tabular}

${ }^{1}$ Assumed in computing the $\mathrm{N}_{2} \mathrm{D}^{+}$total column density in Table 2.

emission is extended and irregular, covering an area of $\sim 40^{\prime \prime}$ in the N-S direction, and $\sim 20^{\prime \prime}$ in the W-E direction. The main emission peak is close to $\mathrm{C} 2$, and the shape of the emission indicates that $\mathrm{N}_{2} \mathrm{H}^{+}(1-0)$ and the $93 \mathrm{GHz}$ continuum are fairlywell overlapping. An extended component in both line and continuum oriented NE-SW roughly corresponds to condensation N. Another compact source, C4, is detected $\sim 20^{\prime \prime}$ south of the map centre. Such a different distribution of the integrated intensity with respect to that of the $\mathrm{N}_{2} \mathrm{H}^{+}(3-2)$ line is probably due to the extended emission being filtered out differently by the two interferometers. To compute the amount of the missing flux, we have compared our interferometric spectra with the single-dish spectra obtained with the IRAM-30 m telescope (see Fontani et al. 2006). We have resampled the single-dish spectra of $\mathrm{N}_{2} \mathrm{H}^{+}(1-0)$ and $(3-2)$ to the same resolution in velocity as the interferometric spectra. In the $\mathrm{N}_{2} \mathrm{H}^{+}(1-0)$ line, the flux measured by the PdBI is $\sim 2$ times less than that measured with the IRAM-30 m telescope, while in the $\mathrm{N}_{2} \mathrm{H}^{+}$(3-2) line the flux measured with the SMA is only one fifth of that measured with the $30 \mathrm{~m}$ antenna. This indicates that the extended emission is much more resolved in the SMA map than in the PdBI map.

\section{2. $\mathrm{N}_{2} \mathrm{H}^{+}$and $\mathrm{N}_{2} \mathrm{D}^{+}$line parameters and deuterium fractionation}

In Table 1 we give the $\mathrm{N}_{2} \mathrm{H}^{+}(1-0)$ and $\mathrm{N}_{2} \mathrm{D}^{+}$(3-2) line parameters of the spectra integrated over the $3 \sigma \mathrm{rms}$ level of the $\mathrm{N}_{2} \mathrm{D}^{+}$ emission in condensations $\mathrm{N}$ and $\mathrm{S}$ : in Cols. 3-7 we list integrated intensity $\left(\int T_{\mathrm{MB}} \mathrm{dV}\right)$, peak velocity $\left(V_{\mathrm{LSR}}\right), F W H M$, opacity of the main component $\left(\tau_{\mathrm{m}}\right)$, and excitation temperature $\left(T_{\mathrm{ex}}\right)$ of the lines. The integrated intensities have been computed over the velocity range given in Col. 2, while for the other parameters we have adopted the fitting procedure described in Sect. 2.1.1 of Fontani et al. (2006). We do not give the line optical depths and $T_{\text {ex }}$ for the $\mathrm{N}_{2} \mathrm{D}^{+}$lines, because in both spectra the uncertainties are comparable to the values obtained. Because of this, we have fitted the lines forcing the optical depth to be 0.1 .

Linewidths and peak velocities of both $\mathrm{N}_{2} \mathrm{H}^{+}$and $\mathrm{N}_{2} \mathrm{D}^{+}$are very similar in both condensations, indicating that they are tracing the same gas. In particular, the lines are $\sim 0.8 \mathrm{~km} \mathrm{~s}^{-1}$ broad for both molecular species, i.e. nearly half of that observed with the IRAM-30m telescope. This could be due either to extended components with different velocities that have been resolved 
out, or to a decrease in turbulence going from the pc-scale to the sub-pc scale. However, it is interesting to notice that the lines are broader than the typical $\mathrm{N}_{2} \mathrm{H}^{+}$and $\mathrm{N}_{2} \mathrm{D}^{+}$lines observed towards low-mass pre-stellar cores, for which values of $\sim 0.2-0.3 \mathrm{~km} \mathrm{~s}^{-1}$ are found on comparable linear scales (Crapsi et al. 2005; Roberts \& Millar 2007).

We have derived $\mathrm{N}_{2} \mathrm{H}^{+}$and $\mathrm{N}_{2} \mathrm{D}^{+}$total column densities, $N\left(\mathrm{~N}_{2} \mathrm{H}^{+}\right)$and $N\left(\mathrm{~N}_{2} \mathrm{D}^{+}\right)$, following the method described in the Appendix of Caselli et al. (2002b), which assumes a constant $T_{\text {ex }} \cdot N\left(\mathrm{~N}_{2} \mathrm{H}^{+}\right), N\left(\mathrm{~N}_{2} \mathrm{D}^{+}\right)$and $D_{\text {frac }}=N\left(\mathrm{~N}_{2} \mathrm{D}^{+}\right) / N\left(\mathrm{~N}_{2} \mathrm{H}^{+}\right)$, derived for $\mathrm{N}$ and $\mathrm{S}$, are listed in Cols. 2, 3 and 4 of Table 2, respectively. These have been obtained using the values of $T_{\mathrm{ex}}$ given in Table 1. For the $\mathrm{N}_{2} \mathrm{D}^{+}$(3-2) lines, we have assumed the excitation temperature of the $\mathrm{N}_{2} \mathrm{H}^{+}(1-0)$ line because we could not derive a reliable value from the fitting procedure when taking into account the line hyperfine splitting, as already pointed out. In both condensations, $D_{\text {frac }}$ is 0.11 , which is comparable to the values of $D_{\text {frac }}$ found in low-mass pre-stellar cores by Crapsi et al. (2005), following the same method. These values are also comparable to those derived by Pillai et al. (2007) in infrareddark clouds from deuterated ammonia. However, their observations are related to the very cold, pc-scale molecular envelope, and not to compact sub-pc scale cores.

\subsection{Nature of the $\mathrm{N}_{2} D^{+}$condensations}

The main finding of this work is that the $\mathrm{N}_{2} \mathrm{D}^{+}$emission in I05345 is concentrated in two condensations, both of them characterised by high values of deuterium fractionation. We now discuss the nature of these condensations.

We have computed the angular diameters of both condensations assuming that the $\mathrm{N}_{2} \mathrm{D}^{+}$integrated intensity profile can be fitted with a 2D Gaussian: the geometric mean of the major and minor axes resulting from these fits were corrected for the beam size. The linear diameters, $L$, were then computed using a source distance of $1.8 \mathrm{kpc}$ (Zhang et al. 2005). The mass of an equivalent homogeneous sphere of diameter $L, M_{\mathrm{N}_{2} \mathrm{H}^{+}}$, was then obtained from $N\left(\mathrm{~N}_{2} \mathrm{H}^{+}\right)$assuming a $\mathrm{N}_{2} \mathrm{H}^{+}$average abundance of $1.5 \times 10^{-10}$ (Fontani et al. 2006). $L$ and $M_{\mathrm{N}_{2} \mathrm{H}^{+}}$are listed in Cols. 5 and 6 of Table 2, respectively. For $\mathrm{N}$ and $\mathrm{S}$, we derive $M_{\mathrm{N}_{2} \mathrm{H}^{+}} \sim 8.7$ and $2.5 M_{\odot}$, and $L \sim 0.09$ and $0.05 \mathrm{pc}$, respectively. These values, together with a $D_{\text {frac }} \simeq 0.1$, suggest that both cores may be low-mass pre-stellar cores. On the other hand, as stated in Sect. 3.2, both $\mathrm{N}$ and $\mathrm{S}$ show lines broader than those typically observed in low-mass pre-stellar cores. We propose three possible scenarios for the nature of the two $\mathrm{N}_{2} \mathrm{D}^{+}$condensations: (i) they represent the residual of the extended cold cloud in which the cluster of young stellar objects was formed, whose physi$\mathrm{cal} / \mathrm{chemical}$ conditions have not been altered yet by the cluster members; (ii) they are a group of unresolved low-mass starless cores; (iii) they are single starless cores, which are either low- to intermediate-mass pre-stellar cores, or the seeds of future massive forming stars.

The first scenario is very unlikely for both $\mathrm{N}$ and $\mathrm{S}$, since the $\mathrm{N}_{2} \mathrm{D}^{+}$(3-2) line is expected to trace gas denser than $\sim 10^{6} \mathrm{~cm}^{-3}$, while the $\mathrm{H}_{2}$ volume densities measured in the pc-scale gas associated with high-mass star formation regions typically reach values no higher than $\sim 10^{4}-10^{5} \mathrm{~cm}^{-3}$. The third scenario is the most probable for $\mathrm{S}$, while for $\mathrm{N}$, which shows an elongated structure (see Fig. 1) indicative of possible multiple low-mass components, the second scenario is also possible. Indeed, the shape of $\mathrm{N}$ resembles that of Oph $\mathrm{A}$, a molecular clump
Table 2. $\mathrm{N}_{2} \mathrm{H}^{+}$and $\mathrm{N}_{2} \mathrm{D}^{+}$total column densities $\left(N\left(\mathrm{~N}_{2} \mathrm{H}^{+}\right)\right.$and $N\left(\mathrm{~N}_{2} \mathrm{D}^{+}\right)$), deuterium fractionation $\left(D_{\text {frac }}\right)$, linear diameter $(L)$, and mass derived from $N\left(\mathrm{~N}_{2} \mathrm{H}^{+}\right)\left(M_{\mathrm{N}_{2} \mathrm{H}^{+}}\right)$for the $\mathrm{N}_{2} \mathrm{D}^{+}$condensations $\mathrm{N}$ and $\mathrm{S}$ of Fig. 1. The uncertainties computed following the standard propagation of the errors are given between parentheses.

\begin{tabular}{cccccc}
\hline \hline Source & $\begin{array}{c}N\left(\mathrm{~N}_{2} \mathrm{H}^{+}\right) \\
\left(\times 10^{13} \mathrm{~cm}^{-2}\right)\end{array}$ & $\begin{array}{c}N\left(\mathrm{~N}_{2} \mathrm{D}^{+}\right) \\
\left(\times 10^{12} \mathrm{~cm}^{-2}\right)\end{array}$ & $D_{\text {frac }}$ & $\begin{array}{c}L \\
(\mathrm{pc})\end{array}$ & $\begin{array}{c}M_{\mathrm{N}_{2} \mathrm{H}^{+}} \\
\left(M_{\odot}\right)\end{array}$ \\
\hline $\mathrm{N}$ & $1.9(0.6)$ & $2.1(0.3)$ & $0.11(0.04)$ & 0.09 & 8.7 \\
$\mathrm{~S}$ & $1.5(0.5)$ & $1.6(0.3)$ & $0.11(0.04)$ & 0.05 & 2.5 \\
\hline
\end{tabular}

with elongated shape, resolved into several low-mass starless cores (André et al. 2007). In this case, the observed broad lines could be simply due to unresolved cores with diffent velocities. A detailed comparison between $\mathrm{N}$ and Oph $\mathrm{A}$ will be done in the forthcoming paper. However, it is also possible that $\mathrm{N}$ is, like $S$, a unique condensation, the observed structure being due to shaping by a powerful outflow associated with another cluster member. In fact, an outflow driven by the massive source $\mathrm{C} 1$ $\left(\sim 20 M_{\odot}\right)$ has been detected by observing the $\mathrm{CO}(2-1)$ line (this line is part of the large dataset obtained with the SMA that will be published in the forthcoming paper), and the red lobe is indeed detected at the edge of condensation $\mathrm{N}$. If both $\mathrm{N}$ and $\mathrm{S}$ are single cores, what could cause the observed broad lines? The larger turbulence could be simply understood as the result of a high pressure environment (see e.g. McKee \& Tan 2002). Despite this, it is not at all clear which fraction of the line width is actually broadened by systematic motions, in particular infall or accretion of molecular material onto the pre-stellar cores. Only high resolution kinematic studies of the large scale gas around these condensations will give us clues on the line width partition, thus providing important constraints on the dynamical evolution of massive star forming regions.

Acknowledgements. It is a pleasure to thank the staff of the Smithsonian Astrophysical Observatory for the SMA observations. We also thank the IRAM staff for their help in the calibration of the PdBI data. Many thanks to the anonymous referee for his/her useful comments and suggestions.

\section{References}

André, Ph., Belloche, A., Motte, F., \& Peretto, N. 2007, A\&A, 472, 519 Beuther, H., Churchwell, E. B., McKee, C. F., \& Tan, J. C. 2007, Protostars and Planets V, 165

Caselli, P., Myers, P. C., \& Thaddeus, P. 1995, ApJ, 455, L77

Caselli, P., Walmsley, C. M., Zucconi, A., et al. 2002a, ApJ, 565, 331

Caselli, P., Walmsley, C. M., Zucconi, A., et al. 2002b, ApJ, 565, 344

Crapsi, A., Caselli, P., Walmsley, C. M., et al. 2005, ApJ, 619, 379

Fontani, F., Caselli, P., Crapsi, A., et al. 2006, A\&A, 460, 709

Ho, P. T. P., Moran, J. M., \& Lo, K. Y. 2004, ApJ, 616, L1

Kuiper, T. B. H., Langer, W. D., \& Velusamy, T. 1996, ApJ, 468, 761

McKee, C. F., \& Tan, J. C. 2002, Nature, 416, 59

Molinari, S., Brand, J., Cesaroni, R., \& Palla, F. 1996, A\&A, 308, 573

Oliveira, C. M., Hébrard, G., Howk, J. C., et al. 2003, ApJ, 587, 235

Pillai, T., Wyrowski, F., Hatchell, J., Gibb, A. G., \& Thompson, M. A. 2007,

A\&A, 467, 207

Qi, C. 2005, The MIR Cookbook

http://cfa-www.harvard.edu/cqi/mircook.html

Roberts, H., \& Millar, T. J. 2007, A\&A, 471, 849

Sault, R. J., Teuben, P. J., \& Wright, M. C. H. 1995, ASPC, 77, 433

Sridharan, T. K., Beuther, H., Schilke, P., Menten, K. M., \& Wyrowski, F. 2002, ApJ, 566, 931

Tafalla, M., Myers, P. C., Caselli, P., Walmsley, C. M., \& Comito, C. 2002, ApJ, 569, 815

Tafalla, M., Santiago, J., Myers, et al. 2006, A\&A, 455, 577

Zhang, Q., Hunter, T. R., Brand, J., et al. 2005, ApJ, 625, 864 